\title{
Heuristics in Legal Decision-Making
}

\author{
Thomas Hoffmann \\ Tallinn Law School, \\ Tallinn University of Technology \\ Akadeemia tee 3, \\ Tallinn 12618, Estonia \\ E-mail:Thomas.hoffmann@taltech.ee
}

\begin{abstract}
With the advent of algorithm-based decisions, generated in the "black boxes" of deep learning ensembles and their controversially discussed ethical and legal implications, the fact that human legal decision-making is to a large degree not based on rational choices, and thus also neither deducible nor predictable, tends to be neglected in legal discussion. This article intends to provide an overview on heuristic patterns in human legal decision-making by pointing out their flaws and benefits, and also provides some advice on how to hedge its risks in legal practice.
\end{abstract}

Keywords: biases, heuristics, legal decision-making, rational choice theory

\section{Introduction}

There are various reasons why 'law on the books' may differ from 'law in action': As the actual practical significance of a legal norm is not determined by the legislator but exclusively by the sum of those applying the law, ${ }^{1}$ that is, the practitioners, it is their cultural imprint, their personal experiences and first of all their acquired habits which mark 'law in action'. As most of these reasons seem to differ individually among all legal practitioners, legal research rarely focusses on eventual patterns behind these differences - although it is not 'law on the books', but 'law in action' which shapes the legal life within our societies. Not all of these reasons can be analysed legally with scientific methods to the fullest; especially as far as 'law in action' can be determined, for example, merely by close personal relations (nepotism) or transfers of values (bribery), research will be

1 On the background, see Clune, 2013. 
basically limited to the comparison of statistics, as the reasons for the divergence from 'law in the books' are too manifold.

However, there is also a rather broad area where the differences between 'law in the books' and 'law in action' are driven by the willingness to apply law correctly and with the best intentions in mind, but it still does not fully follow the logic of the rules presumed by the lawmakers. This area is marked by heuristics, whose patterns are-in contrast to the former-accessible to scientific analysis.

\section{Heuristics in general}

Heuristics is the opposite of logic. ${ }^{2}$ It describes an analytical approach to finding solutions, taking into account a restricted amount of accessible information and assuming conclusions about the system a priori (Wieacker, 1995, p. 1102). These inferred statements may differ from the optimal solution in individual cases but, in sum, provide in daily practice-as heuristic procedures require far less time and effort - a far higher average of accomplishment of all tasks than logically derived findings. Indeed, an optimal result is not always required; often, a "good enough" result may suffice. The result is not deduced but rather "guessed", based on intuition, common sense or the "hunch". The general approach is that a solution is first assumed to be true and then-only as a second step-tried to be set into a general system ("working backwards"). ${ }^{3}$ In natural sciences, typical heuristics are all kinds of trial-and-error-procedures (just as evolution itself, even though lacking the purpose of finding solutions), while in the field of (daily, i.e. non-legal) decision-making the vast majority of decisions is usually based on heuristics. Many of these heuristics may be based on biases, falsifying the concluded results. However, recent research (by Gerd Gigerenzer) has shown that heuristics nevertheless has "certain fast and frugal" (Raab \& Gigerenzer, 2015, p. 1672) properties that are in principle accurate and therefore eliminate most cognitive biases (Gigerenzer et al., 2000). Together with Wolfgang Gaissmaier, Gigerenzer has even claimed that ignoring part of the information available for making a decision, rather than weighing all the options, can lead to more accurate decisions (Gigerenzer \& Gassmaier, 2011). An example of this surprising phenomenon was a query on the future development of some famous joint-stock companies carried out among "ordinary people" in the pedestrian zone of a

On the background, see Bocheński, 1968.

3 On the general concepts of this method in mathematics see more Pólya, 1945. 
major German city. Their prediction about the stock course of these companies turned out to be far more accurate than prior stock market research carried out by a few bank professionals trained for this task, although these "ordinary people" had access to much less information than the stock market professionals. Just their "hunch", "common sense" provided more accurate results than logicbased prediction procedures. But which role do these mechanisms play in law?

\section{Traditional approaches to solving legal problems}

The traditional approach in Western legal systems to solving a legal problem is by forming legal syllogisms, which is done in three steps: The legal problem is distilled from the situation to be solved, a legal norm describing the treatment of a similar situation is determined (may it be from positive law as in continental European systems or rather from case law as in common law), the situation is "subsumed" under the norm, i.e. all conditions provided by the norm are checked against the facts, and the respective findings of this comparison constitute the final result (Fikentscher, 2007, p. 1091). These steps are generally taken not only as the common procedure by judges but also by everybody else applying law.

These four steps are based on logic; the final result is "calculated". The system does not require comprehensive information-logically deduced results can also be achieved with restricted data; legal norms deal with the omnipresent shortage of data with instruments such as sweeping clauses or, for example, procedurally, with the burden of proof. Still, these procedures form-if observed closelyrather a part of 'law on the books', or 'law in academia'. Just as in other fields of life, heuristics plays an important role also in finding (and creating) law, be it with or without intent. A judge in a court of first instance facing a huge workload will usually develop within a short time heuristics on how to cope with his workload; an example is an experienced German judge, who recently confessed to the author:

In fact, for simple claims for compensation for damages, theoretically I do not even have the time to subsume these cases diligently under all respective norms provided by the legislator. I usually read the case carefully, and after many years of practice, I now actually feel immediately after finishing my reading who shall win and how much he should pay. Still, I have to justify my finding with a written reasoning within the judgment, and I tend to look 
for suitable provisions justifying my result rather than deducing the result objectively from a suitable provision. I know that this is not what the system is designed for, but my judgments are much less often appealed against those of many of my colleagues who literally stick to all the details provided for these cases by the legislator, and it saves considerable time to focus on the really complicated cases.

Can this judge-against the background of civil law legal culture-still be considered a "good judge"? Many would say so, although he circumvents the system set up by the legislator. This judge uses heuristics, and one of the purposes of this paper is to find out which heuristics he eventually used, what these heuristics may have been based on, and whether this usage of heuristics in law-which is, no doubt, very common — should be supported or, respectively, how lawmakers should be advised in order to take into account these heuristics.

\section{Heuristics used when applying the law}

Lawmakers assume that those applying their laws apply a rational choice theory (RCT), i.e. that actors will weigh respective consequences provided by legal norms and, based on logical deduction, choose the one that maximizes their advantages or utility. Taking into account that lawmakers do not only intend to provide a set of rules for providing equal justice to all actors bound by it (e.g., most of private law), but also to "provide private incentives to behavior that will maximize social welfare (such as tax law)" and "to directly improve social welfare of a targeted group" (such as public grants) (Korobkin, 2004, p. 2), these aims would be jeopardized if the behavior of the actors bound by these laws does not follow the patterns assumed by the lawmaker.

The following part presents some examples of how actors deviate from the lawmakers' assumptions by not using RCT but heuristic approaches.

\section{Primary addressees (consumers, their lawyers)}

As implied above, heuristics always bear the risk of being based on biases. There are various forms of biases which influence, for example, consumers' choice on how to behave legally in their environment, and many of them are not "fast and frugal" heuristics (leading to a superior accomplishment of all tasks from a broader perspective), but are based on disadvantageous predispositions. Individually 
observed, many of these heuristics seem banal at first, but in accumulation form a rather complex behavioristic constellation which deserves further analysis.

a) Availability bias

A widely used psychological shortcut in practice is the fact that people make judgments about the probability of events with the ease with which examples come to mind. If a party, for example, has read in a newspaper that an insurance company has paid large amounts of money to victims of a natural disaster, he or she may be more inclined to contract with that insurance company, even though he or she does not know about the eventually much higher number of refused payments to other victims (which were not reported in the news). This data may be also available when asked for directly at the company, but as they are not as easily available as the news, they are usually not taken into account.

b) Familiarity bias

Similar to the availability bias, this psychological shortcut applies to various situations in which individuals assume that the circumstances underlying past behavior still hold in the present situation and that the past behavior can thus be correctly applied to the new situation. For example, if a standard contract term has not been proven harmful in previous contracts, it is often assumed that it will not be harmful in the new contract to be signed either, even though the risk may have increased considerably in the meantime.

c) Status quo bias

This bias describes the phenomenon that most people, in general, are reluctant to changes, even though the advantages of another offer are obvious (Samuelson \& Zeckhauser, 1988). For instance, a study carried out in 1991 by Hartmann and Woo (1991) demonstrated that when offered two options for trade-offs between service reliability and rates, $60.2 \%$ of electric power consumers in California preferred to stick to the option designated as their status quo even though the lower rates for respectively lower reliability provided in the second option were economically more advantageous to many in this group. Also, as many as $58.3 \%$ of those in the low-reliability group preferred to stick to the option designated as a status quo, while only $5.8 \%$ decided to switch to the high-reliability option.

d) Escalation of commitment bias

This bias describes the phenomenon that people try to justify increased investment in a decision on the basis of cumulative prior investment, despite 
new evidence suggesting that the cost, starting that day, of continuing the decision outweighs the expected benefit, which people prefer to ignore (in many cases as the decision-maker, consciously or not, does not wish to reveal to others, or herself, that a decision made earlier was wrong). It is somewhat related to the status quo bias, but is so far diverging as in this constellation the actor actively damages her position by investing further in a dead-end-road.

e) Example of a non-biased heuristic

All these examples show how biases may be harmful to heuristic approaches. Still, daily legal practice contains quite a few useful heuristics. Imagine, for instance, that a driver in a hurry uses a highway where the maximum speed is $90 \mathrm{~km} / \mathrm{h}$, and that his or her goal is not primarily to comply with the law but to minimize the costs, including those of eventual speeding tickets (Goldstein, 2006, p. 451). An RCT approach would have to take into account criteria such as the cost of speeding tickets during the year, the time spent traveling, the cost of a speeding ticket, the number of tickets already received (as the costs may increase for repeated offenses), the likelihood of accidents or the weather conditions, the interest to possibly avoid any fatal accidents categorically, the driver's own experiences with the probability of police presence on this road and his or her knowledge about eventual hiding places, the driver's degree of enjoyment of driving fast, his or her state of boredom because of boring roads/environment, absence of company, etc. In a logical approach, all these criteria would have to be calculated and finally processed towards choosing the advisable speed. In fact, most drivers apply a heuristic approach: First, they check whether other cars are exceeding the speed limit as well, in which case they simply follow them at the same speed. If there are no other cars present, many drivers tend to exceed the speed limit by about $15 \mathrm{~km} / \mathrm{h}$. In fact, in practice this procedure quite likely will lead to no- or low-cost speed tickets; although it is not optimal (as the risk of receiving a speed ticket may still substantiate from time to time), it serves, with minimum computation efforts, the previously set-up goal sufficiently. 


\section{Secondary addressees (judges)}

Although legally trained, also judges are generally subject to biases such as those described above. However, there are other biases which are even more relevant when it comes to making a legal decision.

a) Hindsight bias

This bias describes the phenomenon that, given the factual outcome of a development, the probability seems much higher for this outcome than for others, if seen ex post. At court, this bias may result in defendants being judged as capable of preventing a bad outcome: for example, in cases where there is an assumption of risk, hindsight bias may contribute to judges perceiving the event as even riskier merely due to the poor outcome. This may lead the judge/jury to feel that the plaintiff should have exercized greater caution in the situation, although, if an a priori perspective is taken, this caution would not have been deemed as necessary (Steinbeck \& Lachenmaier, 2014, p. 2090).

b) Anchoring and adjustment

This, in practice, no less important bias describes the tendency to rely too heavily on the first piece of information offered (the "anchor") when making decisions. For example, if the state attorney suggests a certain period of imprisonment for the accused, the judge will be automatically influenced by this "anchor" when determining his proposition. Various experiments have shown that this benchmark effect occurs even when the judge is proposed a certain number by persons with no legal training.

\section{Consequences}

\section{For lawmakers}

As a form of heuristic procedure in practice, the backbone of most daily decisions we make, it is essential that the lawmaker becomes aware of these mechanismsand especially of the various biases to which we are exposed. As law also aims to steer society towards peace and welfare, it should be written in a way which takes into account the actual behavioural patterns of those acting under the law. This requires a detailed study of heuristic patterns in society, including their implied biases, which ought to be made accessible to all institutions involved in lawmaking. If too many personal decisions are made by heuristics based on biases, 
the degree of satisfaction of those bound by these, at the time disadvantageous decisions will decrease, having a negative impact on the trust in law in general, or inducing purely social mechanisms to reach one's personal goals effectively beyond the law. This can be done by providing regulations which try to prevent the use of biases, which is already widely practiced in the field of consumer protection law (e.g., the duty of financial service providers, such as banks, etc., to provide a prospectus naming in detail all the risks of the respective investment, preventing the availability bias). But lawmakers shall not only prevent the use of biases, they can also make use of them in order to reach specific legislative purposes: If the aim is to provide health insurance to all citizens, the lawmaker can make use of the status quo bias by providing everybody health insurance policy by default, supplementing this model with an opt-out-mechanism for those who want to save on insurance fees. This model, for example, is used by the churches in Germany, to which members belong automatically after baptism, thus being subject to church taxes once they are grown up.

\section{For judges}

Judges, first of all, should be aware of the fact that they are generally no less affected by heuristic patterns than legally non-trained people, and that various biases influence their decisions. When it comes to bias-free "fast and frugal" heuristics in the sense of Gigerenzer, there may indeed be quite a broad practice of apparently well-functioning heuristic patterns among judges in Western legal systems. While heuristics have generally been proven as a useful tool in other fields of solution-finding, a heuristic approach practiced by judges remains highly problematic: The central problem is not that judgments based on heuristics in general would be automatically less fair, but rather that they become unforeseeable. Legal training_and all jurisprudence-in Western legal systems is based on trying to predict a judge's decision by applying the same steps that he or she does (Fikentscher, 2007, p. 1105). These steps can only be resumed once the judge is guided by rational, i.e. logical rules; if it is common sense or his "hunch" (whatever the fairness of the result), the reason to study law at all ceases to exist. A judge's decision must remain logically deductive; otherwise, any legal reasoning loses its function. The German judge mentioned above, therefore, may be a good judge in practice, but she should be ready to have a coherent logicbased justification for her judgment at hand, once she is asked for it. The system practiced by her cannot replace the logical-deductive patterns of solution-finding developed in Western legal systems. 


\section{Conclusion}

Heuristics, at first sight, seem to have much in common with legal pragmatism or legal realism (Rechtsrealismus), as it was en vogue in the 1920s (Fikentscher, 2007 , p. 1100). While the unforeseeability problem is an essential part of the heuristic approach, today it is no longer controversial that heuristics cannot replace the traditional logical-deductive approach which has marked Western European systems since the antiquity, when it comes to judicature. As heuristics nevertheless play an important role wherever decisions are made, and as most of these heuristics are not "good", i.e. "fast and frugal" heuristics, they must not be ignored by sticking to the assumption that people make rational choices (i.e., follow RCTs). This awareness has partly already been implemented by lawmakers, who either try to prevent biases among actors or even actively in the form of "nudging" (Hansen, 2015) or "cautious paternalism" (Korobkin, 2004, p. 16), or to make use of these biases by creating heuristic-based incentives towards a behaviour socially envisaged by lawmakers. Judges, on the other hand, first of all, shall be aware that they are generally at risk of following biases just like legally non-trained decision-makersespecially if their workload makes them prefer heuristic approaches rather than the diligent but far more time-consuming logical-deductive approach by classically subsuming the facts of the case under the text of the law. But even if their judgments have been proceeded by non-biased heuristics, they should remember that there is always a sound "logical base" at hand which justifies the decision based on effective law, as their reasoning must remain coherent towards the parties and other judges.

\section{References}

Bocheński, J. M. (1968), Ancient Formal Logic, 4th ed., Amsterdam: North Holland Publishing Company.

Clune, B. (2013), 'Law in action and law on the books: A primer', [Blog entry] New Legal Realism Conversations, May 2013. Retrieved from http://newlegalrealism.wordpress. com/2013/06/12/law-in-action-and-law-on-the-books-a-primer/ [accessed $20 \mathrm{Feb}$ 20201

Fikentscher, W. (2007), 'Juristische Heuristik?' in Festschrift für Claus-Wilhelm Canaris zum 70. Geburtstag, München: C. H. Beck, vol. 2, pp. 1091-1106.

Gigerenzer, G. (1991), 'How to make cognitive illusions disappear: beyond "heuristics and biases", European Review of Social Psychology, vol. 2, no. 1, pp. 83-115. https://doi.org/10.1080/14792779143000033 
Gigerenzer, G. \& Gaissmaier, W. (2011), 'Heuristic decision making,' Annual Review of Psychology, vol. 62, pp. 451-482.

https://doi.org/10.1146/annurev-psych-120709-145346

Gigerenzer, G.; Todd, P. M. \& ABC Research Group (2000), Simple Heuristics That Make Us Smart, Oxford: Oxford University Press.

Goldstein, D. et al. (2006), 'Group Report: How do heuristics mediate the impact of law on behavior?' in G. Gigerenzer \& C. Engel (eds.) Heuristics and the Law, Cambridge, MA: MIT Press, pp. 439-367.

Hansen, P. G. (2015), 'Nudge and libertarian paternalism: Does the hand fit the glove?' European Journal of Risk Regulation, vol. 1, no. 1, pp. 155-174. https://doi.org/10.1017/S1867299X00005468

Hartman, R. S.; Doane, M. J. \& Woo, C. -K. (1991), 'Consumer rationality and the status quo,' Quarterly Journal of Economics, vol. 106, no. 1, pp. 141-162. https://doi.org/10.2307/2937910.

Korobkin, R. B. (2004), The Problems with Heuristics for Law, University of California, Los Angeles School of Law, Law \& Economics Research Paper Series, Research Paper no. 04-1. https://doi.org/10.2139/ssrn.496462

Pólya, G. (1945), How to Solve It: A New Aspect of Mathematical Method, Princeton, NJ: Princeton University Press. https://doi.org/10.1515/9781400828678

Raab, M. \& Gigerenzer, G. (2015), 'The power of simplicity: a fast-and-frugal heuristics approach to performance science,' Frontiers in Psychology, vol. 6, p. 1672. https://doi.org/10.3389/fpsyg.2015.01672

Samuelson, W. \& Zeckhauser, R. (1988), 'Status quo bias in decision making,' Journal of Risk and Uncertainty, vol. 1, pp. 7-59. https://doi.org/10.1007/BF00055564

Steinbeck, A. \& Lachenmaier, A. (2014), 'Verhaltensökonomik im Gerichtssaal,' Neue Juristische Wochenschrift, vol. 29/2014, pp. 2086-2091.

Wieacker, F. (1995), A History of Private Law in Europe, Oxford: Oxford University Press.

Dr.iur. Thomas Hoffmann, LL.M., is professor of private law at Tallinn Law School at Tallinn University of Technology, Estonia. He graduated and obtained his PhD in law at the University of Heidelberg. After his admission to the German bar, he worked as an associated lawyer for Noerr LLP in their Kyiv and Berlin offices, as a research fellow at the Institute of East European Law in Kiel, Germany, and as DAAD lecturer in law at the University of Tartu, Estonia. Hoffmann's research focuses on comparative private law, here especially insolvency law, private international law, contracts in digital environments, consumer law and regional legal history. 